\title{
Comparison of methylation capture sequencing and Infinium MethylationEPIC array in peripheral blood mononuclear cells
}

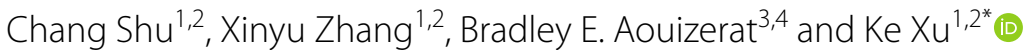

\begin{abstract}
Background: Epigenome-wide association studies (EWAS) have been widely applied to identify methylation CpG sites associated with human disease. To date, the Infinium MethylationEPIC array (EPIC) is commonly used for highthroughput DNA methylation profiling. However, the EPIC array covers only $30 \%$ of the human methylome. Methylation Capture bisulfite sequencing (MC-seq) captures target regions of methylome and has advantages of extensive coverage in the methylome at an affordable price.

Methods: Epigenome-wide DNA methylation in four peripheral blood mononuclear cell samples was profiled by using SureSelectXT Methyl-Seq for MC-seq and EPIC platforms separately. CpG site-based reproducibility of MC-seq was assessed with DNA sample inputs ranging in quantity of high (> $1000 \mathrm{ng}$ ), medium (300-1000 ng), and low (150 ng-300 ng). To compare the performance of MC-seq and the EPIC arrays, we conducted a Pearson correlation and methylation value difference at each CpG site that was detected by both MC-seq and EPIC. We compared the percentage and counts in each CpG island and gene annotation between MC-seq and the EPIC array.
\end{abstract}

Results: After quality control, an average of 3,708,550 CpG sites per sample were detected by MC-seq with DNA quantity $>1000 \mathrm{ng}$. Reproducibility of DNA methylation in MC-seq-detected CpG sites was high among samples with high, medium, and low DNA inputs ( $r>0.96)$. The EPIC array captured an average of $846,464 \mathrm{CpG}$ sites per sample. Compared with the EPIC array, MC-seq detected more CpGs in coding regions and CpG islands. Among the 472,540 $\mathrm{CpG}$ sites captured by both platforms, methylation of a majority of CpG sites was highly correlated in the same sample $(r$ : 0.98-0.99). However, methylation for a small proportion of CpGs $(N=235)$ differed significantly between the two platforms, with differences in beta values of greater than 0.5 .

Conclusions: Our results show that MC-seq is an efficient and reliable platform for methylome profiling with a broader coverage of the methylome than the array-based platform. Although methylation measurements in majority of CpGs are highly correlated, a number of CpG sites show large discrepancy between the two platforms, which warrants further investigation and needs cautious interpretation.

Keywords: Methylation capture sequencing, EPIC, DNA methylation, Peripheral blood mononuclear cells

*Correspondence: ke.xu@yale.edu

${ }^{1}$ Department of Psychiatry, Yale School of Medicine, New Haven, CT 06516, USA

Full list of author information is available at the end of the article

\section{Introduction}

The rapid increase in the number of epigenome-wide association studies (EWAS) have successfully identified differentially methylated CPG sites that are associated with environmental exposures and diseases [1-6]. Such DNA methylation marks have been used as biomarkers for diagnosing, subtyping, and monitoring disease 
progression [7-11]. The most popular and affordable methods to profile epigenome-wide DNA methylation are array-based platforms, primarily the Illumina Human Methylation $450 \mathrm{~K}(450 \mathrm{~K})$ and Infinium MethylationEPIC (EPIC) BeadChips (Illumina Inc, San Diego, CA). These arrays utilize Illumina's beadchip technology that does not require polymerase chain reaction (PCR), but is subject to dye intensity biases between the two platforms [12]. These arrays have limited coverage of the methylome and can only detect up to 870,000 CpGs across the epigenome, leaving a large proportion of $\mathrm{CpG}$ sites unmeasured. Moreover, the EPIC array offers improved but still suboptimal coverage of regulatory elements [13]. Whole-genome bisulfite sequencing (WGBS) is able to capture more than 28 million CpGs, but the feasibility remains low for the population-based EWAS due to high cost and large genomic DNA input requirements to compensate for degradation during DNA bisulfite treatment. Alternatively, Methylation Capture Sequencing (MC-seq) is able to detect DNA methylation at singlenucleotide resolution utilizing a targeted next-generation sequencing approach [14]. It permits profiling of significantly more $\mathrm{CpG}$ sites than the EPIC array, requires less genomic DNA input than WGBS, and less expensive than WGBS, but can be susceptible to bias due to the presence of PCR duplicates. Feature-to-cost comparisons among different platforms can help understand the utilities of each platform and provide guidance for investigators in choosing a methylation profiling platform.

A few studies have compared the $\mathrm{CpG}$ coverage, reproducibility, and performance of array-based and MC-seq platforms [15-17]. Teh et al. compared MC-seq and the $450 \mathrm{~K}$ array in seven DNA samples extracted from saliva [15]. A recent study compared the EPIC array and TruSeq targeted bisulfite sequencing in four cord blood DNA samples [17]. However, no comparisons of MC-seq and array-based methylome profiling of peripheral blood mononuclear cells (PBMCs) has been reported. Here, we profiled the DNA methylome in PBMCs using the Agilent SureSelect Methyl-Seq platform and compared the results to the EPIC array in DNA samples extracted from PBMCs.

\section{Methods}

\section{Methylation capture sequencing (MC-seq)}

\section{DNA samples description}

DNA was extracted from de-identified PBMCs collected from four individuals. Genomic DNA quality was determined by estimating the A260/A280 and A260/ A230 ratios by spectrophotometry and concentration by fluorometry. DNA integrity and fragment size were confirmed using a microfluidic chip run on an Agilent Bioanalyzer. To assess the reproducibility of MC-seq by
DNA quantity, DNA samples from each participant were profiled in triplicate times with high ( $>1000 \mathrm{ng}$ ), medium (300-1000 ng), and low (150-300 ng) DNA input. In total, 12 DNA samples were measured by MC-seq. Bisulfate conversion was conducted for each DNA sample as described below.

\section{Methyl-seq target enrichment library prep}

Indexed paired-end whole-genome sequencing libraries were prepared using the SureSelect XT Methyl-Seq kit (Agilent, part\#G9651B). Genomic DNA was sheared to a fragment length of 150-200 bp using focused acoustic energy delivered by the Covaris E220 system (Covaris, part\#500003). Fragmented sample size distribution was determined using the Caliper LabChip GX system (PerkinElmer, Part\#122000). Fragmented DNA ends were repaired with T4 DNA Polymerase and Polynucleotide Kinase and "A" base was added using Klenow fragment in a single reaction followed by AMPure XP bead-based purification (Beckman Coulter, part\#A63882). The methylated adapters were ligated using T4 DNA ligase followed by AMPure XP bead purification. Quality and quantity of adapter-ligated DNA were assessed using the Caliper LabChip GX system. Samples yielding >350 ng were enriched for targeted methylation sites by using the custom SureSelect Methyl-Seq Capture Library. Hybridization was performed at $65{ }^{\circ} \mathrm{C}$ for $16 \mathrm{~h}$ using a C1000 Thermal Cycler (BIO-RAD, part\# 1851197). Once the enrichment was completed, the samples were mixed with streptavidin-coated beads (Thermo Fisher Scientific, part\#65602) and washed with a series of buffers to remove non-specific bound DNA fragments. DNA fragments were eluted from beads with $0.1 \mathrm{M} \mathrm{NaOH}$. Unmethylated $C$ residues of enriched DNA were modified by bisulfite conversion using the EZ DNA Methylation-Gold Kit (Zymo Research, part\#D5005). The SureSelect enriched, bisulfite-converted libraries were PCR amplified using custom-made indexed primers (IDT, Coralville, Iowa). Dual-indexed libraries were quantified by quantitative polymerase chain reaction (qPCR) using the Library Quantification Kit (KAPA Biosystems, Part\#KK4854) and inserts size distribution was assessed using the Caliper LabChip GX system. Samples with a yield of $\geq 2 \mathrm{ng} / \mu \mathrm{l}$ were proceeded to sequencing.

\section{Flow cell preparation and sequencing}

Sample concentrations were normalized to $10 \mathrm{nM}$ and loaded onto an Illumina NovaSeq flow cell at a concentration that yields 40 million passing filter clusters per sample. Samples were sequenced using $100 \mathrm{bp}$ paired-end sequencing on an Illumina HiSeq NovaSeq according to Illumina standard protocol. The $10 \mathrm{bp}$ dual index was read during additional sequencing reads that 
automatically follows the completion of the first read. Data generated during sequencing runs were simultaneously transferred to the Yale Center for Genome Analysis high-performance computing cluster. A positive control (prepared bacteriophage Phi X library) provided by Illumina was spiked into every lane at a concentration of $0.3 \%$ to monitor sequencing quality in real time.

\section{Preprocessing and quality control}

Signal intensities were converted to individual base calls during a run using the system's Real Time Analysis (RTA) software. Sample de-multiplexing and alignment to the human genome was performed using Illumina's CASAVA 1.8.2 software suite. The sample error rate was required to be less than $1 \%$ and the distribution of reads per sample in a lane was required to be within reasonable tolerance.

Quality control (QC) on MC-seq was conducted following standard procedure as previously described [18]. Quality of sequence data was examined by using FastQC (ver. 0.11.8). Adapter sequences and fragments at $5^{\prime}$ and $3^{\prime}$ (phred score $<20$ ) with poor quality were removed by Trim_galore (ver. 0.6.3_dev). We used Bismark pipelines (ver. v0.22.1_dev) to align the reads to the bisulfite human genome (hg19) with default parameters [19]. Qualitytrimmed paired-end reads were transformed into a bisulfite converted forward strand version $(\mathrm{C} \rightarrow \mathrm{T}$ conversion) or into a bisulfite-treated reverse strand $(\mathrm{G} \rightarrow \mathrm{A}$ conversion of the forward strand). Duplicated reads were removed from the Bismark mapping output by deduplicate_bismark and $\mathrm{CpG}, \mathrm{CHG}$, and $\mathrm{CHH}$ (where $H=\mathrm{A}, \mathrm{T}$, or C) were extracted by bismark_methylation_extractor.

All CpG sites were grouped by sequencing coverage, also known as read depth. The groups with coverage of $1 \times$ to $100 \times$ were used to test the relationship between coverage and number of $\mathrm{CpG}$ sites. Only the $\mathrm{CpG}$ sites with coverage $>10 \times$ depth were used for final comparisons to ensure MC-seq data quality. Genes were annotated using Homer annotatePeaks.pl, including intergenic, 5'UTR, promoter, exon, intron, $3^{\prime} \mathrm{UTR}$, transcription start site (TTS), and non-coding categories. CpG island, shore, shelf, and open sea annotation were defined by locally developed bash and $\mathrm{R}$ scripts based on genomic coordinates (hg19) of CpG islands from the UCSC genome browser. CpG shores was defined as up to $2 \mathrm{~kb}$ from $\mathrm{CpG}$ islands and $\mathrm{CpG}$ shelf was defined as up to $2 \mathrm{~kb}$ from a CpG shore.

\section{Assessment of reproducibility}

We assessed CpG- and participant-based reproducibility for MC-seq among 12 samples with DNA quantity of high, medium, and low input in two ways. First, CpGbased reproducibility was assessed by calculating Pearson correlations using the $\mathrm{CpG}$ sites in common of the samples from the same participant with different input DNA quantities. Scatterplots were rendered showing 10,000 randomly selected common $\mathrm{CpG}$ sites comparing samples with high and medium, high and low, and medium and low DNA inputs. Second, participant-based reproducibility was assessed by comparing methylation profiles among pairs of participants using the samples with high DNA inputs, by calculating Pearson correlations of common CpG sites.

\section{EPIC array data preprocessing}

The Infinium MethylationEPIC array (Illumina, San Diego, CA, USA) was used to measure PBMC DNA methylation profiles from the same four participants. These four samples with DNA input of $1000 \mathrm{ng}$ were preprocessed using standard procedures as previously described [20]. Briefly, the predicted sex based on methylome was consistent with self-reported sex for all samples. All samples had a call rate greater than 0.15. A total of 19,090 CpG sites on X chromosomes and 537 CpG sites on $Y$ chromosomes were filtered. A total of 846,464 CpG sites passed quality control.

\section{Comparison of methylation at each $\mathrm{CpG}$ site between MC-seq and EPIC array}

The overall distribution of gene annotation in relation to $\mathrm{CpG}$ island and genetic region between MC-seq and EPIC array data from the four participants was compared. Common CpG sites between MC-seq and EPIC array assays were defined according to genomic coordinates. Pearson correlation and the absolute beta-difference value $(\Delta \beta)$ were calculated among common CpG sites between MC-seq methylation percentage values and EPIC methylation beta values by using $\mathrm{R}$ (ver. 3.5.1). If median $\Delta \beta$ of the common CpG site between two platforms was $>0.1$, it was defined as a discordant $\mathrm{CpG}$ pair; otherwise, the $\mathrm{CpG}$ site was defined as a concordant CpG pair. The density plot of $\Delta \beta$ and a Manhattan plot showing the distribution of $\Delta \beta$ across epigenome were illustrated. Scatterplots were rendered showing the correlation of $\beta$ values from 10,000 randomly selected $\mathrm{CpG}$ sites measured by both MC-seq and EPIC array.

\section{Results}

\section{MC-seq overview and reproducibility}

In MC-seq, all sequences were efficiently mapped to the reference genome with greater than $89 \%$ mapping efficiency. Interestingly, the number of non-CpG sites was significantly greater than the number of $\mathrm{CpG}$ sites. Among all detected methylation sites by MC-seq, 11\% were $\mathrm{CpG}$ sites, $65 \%$ were $\mathrm{CHH}$ sites, and $24 \%$ were $\mathrm{CHG}$ sites (Fig. 1a). 
Figure $1 \mathrm{~b}$ shows the relationship of the number of detected CpG sites and depth of sequence coverage by MC-seq in one sample. The depth of read at which the majority of sites were sequenced was estimated to be approximately $10 \times$ coverage, observed as the inflection point of the distribution of Fig. 1b. An increase of depth only slightly increased the capture of CpG sites and the inflection point is on $10 \times$ coverage, consistent with previous literature $[15,17]$. Thus, the number of $\mathrm{CpG}$ sites with coverage $\geq 10 \times$ from $M C$-seq was used in subsequent analyses.

After quality control filtering, MC-seq captured an average of 2,878,207 methylation CpG sites with coverage $\geq 10 \times$ among the 12 DNA samples, with an average of 3,708,550 CpG sites among samples with high
DNA input (>1000 ng), an average of 3,046,172 CpG sites among samples with medium DNA input (300$1000 \mathrm{ng}$ ), and an average of $1,879,898 \mathrm{CpG}$ sites among samples with low DNA input (150-300 ng) (Fig. 1c and Table 1). Despite the fact that the detected number of $\mathrm{CpG}$ sites varied depending on DNA input quantity, CpG-based correlation among the common $\mathrm{CpG}$ sites between samples with high and medium, high and low DNA input quantities exceeded $r>0.95$. Correlations of common $\mathrm{CpG}$ sites between medium and low DNA inputs were also high with $r$ in $0.92-0.94$ (Table 2). Figure 1d shows the scatterplot of 10,000 randomly selected common CpGs between samples with high and medium, high and low, and medium and low DNA input quantities. Pair-wise participant-based
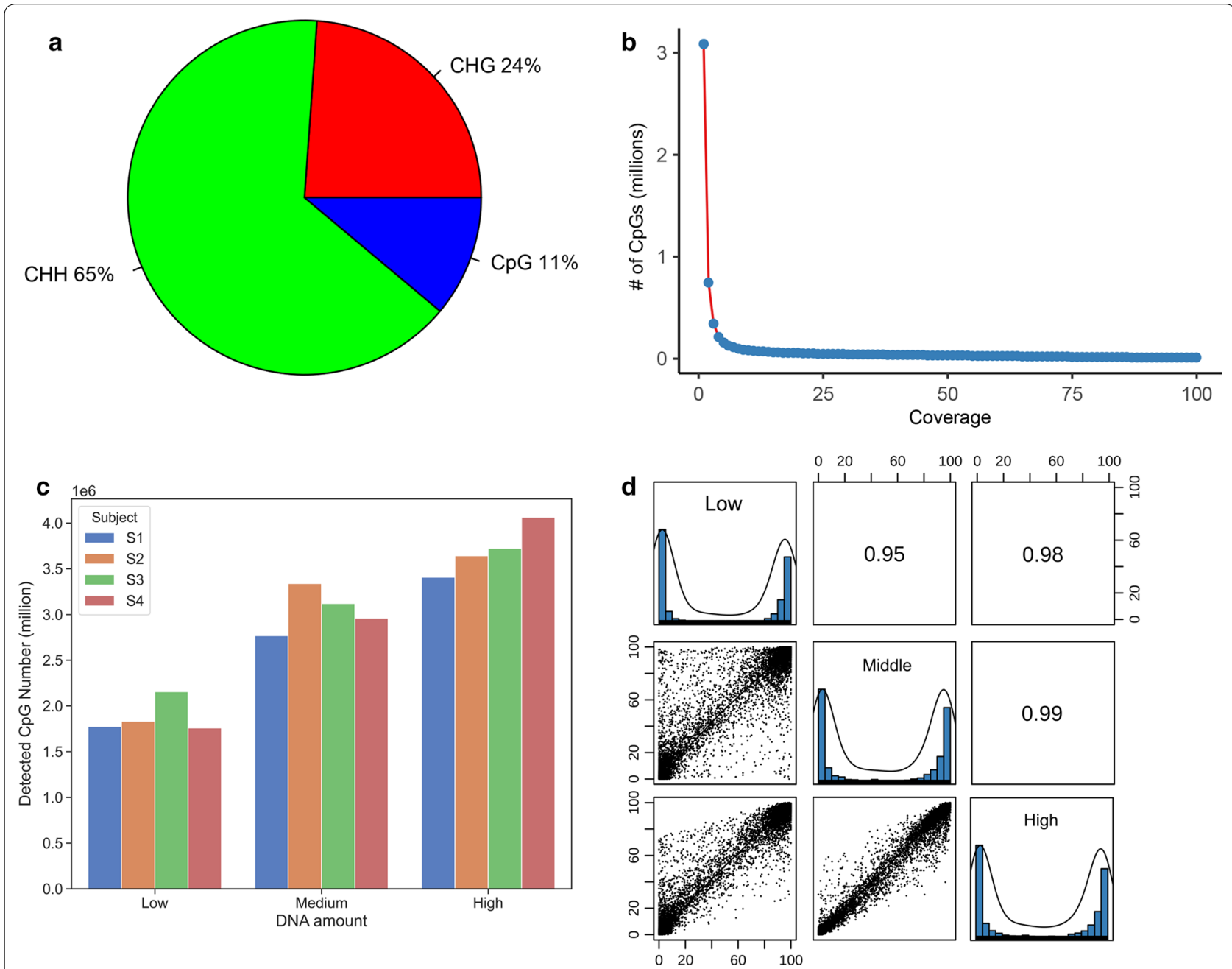

Fig. 1 Methylation Capture Sequencing (MC-seq). a Distribution of methylation sequence context (CpG, CHH, CHG); b Coverage depth versus a number of detected CpG sites; c Detected CpG sites in low, medium, and high DNA inputs for four participants using MC-seq with minimum coverage $\geq 10 x ; \mathbf{d}$ Scatterplots comparing 10,000 randomly selected common CpG sites among samples with high, medium, and low DNA input quantities and their Pearson correlations 
Table 1 Detected CpG number by DNA amount in MC-seq with coverage $\geq 10 x$

\begin{tabular}{llll}
\hline DNA amount & Participant ID & CpG number & $\begin{array}{l}\text { Average } \\
\text { CpG } \\
\text { number }\end{array}$ \\
\hline Low & S1 & $1,774,940$ & $1,879,898$ \\
& S2 & $1,831,086$ & \\
Medium & S3 & $2,154,732$ & \\
& S4 & $1,758,834$ & \\
& S1 & $2,768,456$ & $3,046,172$ \\
High & S2 & $3,338,200$ & \\
& S3 & $3,119,259$ & \\
& S4 & $2,958,772$ & \\
& S1 & $3,406,879$ & $3,708,550$ \\
Total average & S2 & $3,642,776$ & \\
\hline
\end{tabular}

correlations were high as $r>0.98$ among common CpG sites (Table 3). Overall, MC-seq exhibited good reproducibility. The methylation profile generating in high DNA input from each participant was used for subsequent analyses.
Distribution of methylome regions by MC-seq and EPIC

We compared genome-wide DNA methylation captured by MC-seq and by EPIC array in the four high DNA input samples. An average of 3,708,550 CpG sites were detected by MC-seq and 846,464 CpG sites by EPIC array. Overall, MC-seq detected 11.5 times more $\mathrm{CpG}$ sites in exons and 10.2 times more $\mathrm{CpG}$ sites in $5^{\prime}$ UTR region compared to the EPIC array, and 4.8 to 8.9 times more $\mathrm{CpG}$ site in other categories of genomic regions by $\mathrm{MC}$-seq compared to EPIC array. However, the proportion of CpGs out of all CpGs successfully measured that map to gene regions in MC-seq as compared to the EPIC array did not significantly differ between these two platforms. For example, the proportion of CpG sites in transcription termination site (TTS) regions was similar between two platforms. MC-seq showed slightly greater proportions of CpG sites in $5^{\prime} \mathrm{UTR}$ and exon regions, while the EPIC array detected a greater proportion of $\mathrm{CpG}$ sites in promoter regions (Fig. 2a). In terms of $\mathrm{CpG}$ sites in relation to $\mathrm{CpG}$ islands including open seas, shelves, and shores, MC-seq detected 10.9 times more CpG sites located on CpG islands and 5.4-6.2 times more on other regions compared with the EPIC array. The proportion of $\mathrm{CpG}$ islands detected by MC-seq was greater than by the EPIC array (42\% versus $29 \%$ ), while the EPIC array detected a

Table 2 Comparison of MC-seq between samples with high, medium, and low DNA input amount

\begin{tabular}{|c|c|c|c|c|}
\hline \multirow[t]{2}{*}{ Participant ID } & \multicolumn{4}{|c|}{ DNA amount } \\
\hline & High & Medium & Common CpG & $\begin{array}{l}\text { Pearson } \\
\text { correlation }\end{array}$ \\
\hline S1 & $3,406,879$ & $2,768,456$ & $2,747,844$ & 0.984 \\
\hline S2 & $3,642,776$ & $3,338,200$ & $3,283,296$ & 0.984 \\
\hline S3 & $3,722,552$ & $3,119,259$ & $3,101,938$ & 0.977 \\
\hline \multirow[t]{3}{*}{ S4 } & $4,061,994$ & $2,958,772$ & $2,957,239$ & 0.979 \\
\hline & \multicolumn{4}{|c|}{ DNA amount } \\
\hline & High & Low & Common CpG & $\begin{array}{l}\text { Pearson } \\
\text { correlation }\end{array}$ \\
\hline S1 & $3,406,879$ & $1,774,940$ & $1,771,936$ & 0.960 \\
\hline S2 & $3,642,776$ & $1,831,086$ & $1,829,919$ & 0.966 \\
\hline S3 & $3,722,552$ & $2,154,732$ & $2,153,175$ & 0.974 \\
\hline \multirow[t]{3}{*}{ S4 } & $4,061,994$ & $1,758,834$ & $1,758,622$ & 0.963 \\
\hline & \multicolumn{4}{|c|}{ DNA amount } \\
\hline & Medium & Low & Common CpG & $\begin{array}{l}\text { Pearson } \\
\text { correlation }\end{array}$ \\
\hline S1 & $2,768,456$ & $1,774,940$ & $1,745,241$ & 0.942 \\
\hline S2 & $3,338,200$ & $1,831,086$ & $1,827,536$ & 0.943 \\
\hline S3 & $3,119,259$ & $2,154,732$ & $2,135,980$ & 0.939 \\
\hline S4 & $2,958,772$ & $1,758,834$ & $1,744,416$ & 0.928 \\
\hline
\end{tabular}


Table 3 Overlap of detected CpG across samples with high DNA input amount by MC-seq

\begin{tabular}{llll}
\hline Participant ID 1 & Participant ID 2 & Common CpG & Pearson $\boldsymbol{R}$ \\
\hline S1 & S2 & $3,336,037$ & 0.980 \\
S1 & S3 & $3,350,314$ & 0.976 \\
S1 & S4 & $3,394,970$ & 0.982 \\
S2 & S3 & $3,519,772$ & 0.978 \\
S2 & S4 & $3,613,753$ & 0.982 \\
S3 & S4 & $3,676,406$ & 0.978 \\
\hline
\end{tabular}

modestly higher percentage of $\mathrm{CpG}$ sites located in open seas than the MC-seq (39\% versus 31\%) (Fig. 2b).

\section{Comparison of Common CpG sites Measured by MC-seq and EPIC}

A total of $472,540 \mathrm{CpG}$ sites were measured by both platforms. Overall, the correlations of these shared CpG sites was high, ranging from $r=0.983$ to 0.985 across the four samples (Fig. 3a). Figure $3 \mathrm{~b}$ presents the distribution of the absolute difference of methylation $\beta$ values between MC-seq and EPIC. A small proportion of CpG sites (1.4\%) were discordant (i.e., $\Delta \beta>0.1$ ), while $98.6 \%$ of $\mathrm{CpG}$ sites were concordant (i.e., $\Delta \beta<0.1$ ). Figure 3a presents the concordant (blue) and discordant $\mathrm{CpG}$ sites (green) between MC-seq and EPIC for participant S1 (Fig. 3a). The 60,753 discordant CpG sites appeared to be randomly distributed across the epigenome (Additional file 1: Figure S1). Among the discordant $\mathrm{CpG}$ sites, we identified 239 CpG sites with highly discrepant methylation (i.e., $\Delta \beta>0.5$ ) (Table 4). Addition file 2: Table S1 presents top 100 discordant $\mathrm{CpG}$ sites with medium discrepant methylation $(\mathrm{D} \beta=0.1 \sim 0.4)$ Additional file 3: Figure S2 shows that participants S2, S3, and S4 have similar distribution of concordant and discordant plots as participant S1.

Density plots of methylation $\beta$ showed bimodal distribution using both the MC-seq and the EPIC array platforms (Fig. 3c). Density of methylated $\mathrm{CpG}$ sites was slightly higher than the density of unmethylated $\mathrm{CpG}$ sites on both platforms. However, the two peaks in the EPIC array density plot were closer than the two peaks in the MC-seq density plot (Fig. 3c), indicating that MCseq captures a higher dynamic range (i.e., more methylated and unmethylated) of CpG sites than the EPIC array. Additional file 4: Figure S3 shows that participants S2, S3, and $\mathrm{S} 4$ have similar density plots.

\section{Discussion}

We profiled the same PBMC samples using the MC-seq and EPIC array platforms and compared their performance. Our results show that the Agilent SureSelect Methyl-Seq targeted enrichment platform produced high-quality DNA methylation sequencing data at single base-pair resolution. MC-seq can reliably detect CpG sites with DNA input quantities as low as $300 \mathrm{ng}$. Overall, MC-seq detected 3-4 times more CpG sites
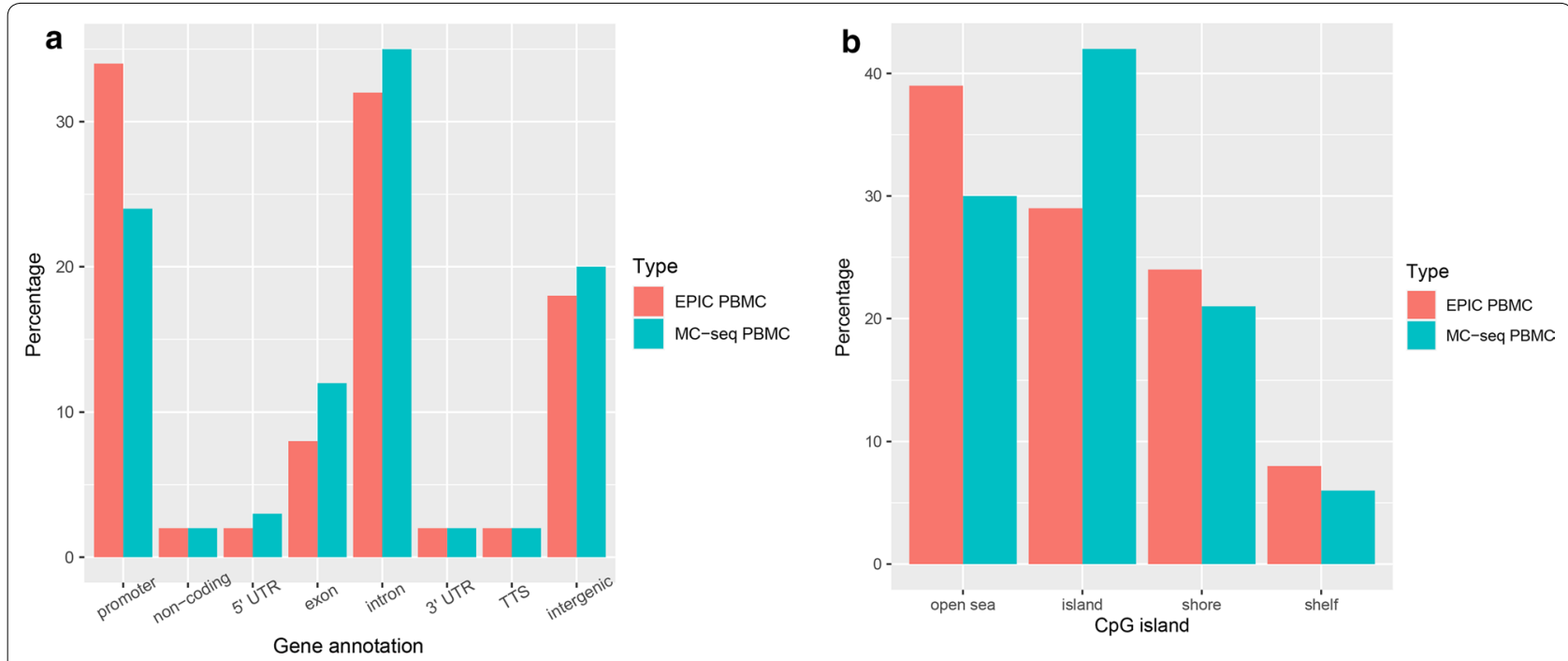

Fig. 2 Comparison of CpG proportion in epigenomic regions between MC-seq and EPIC. a Distribution of genomic regions (intergenic, promoter, $5^{\prime}$ UTR, exon, intron, non-coding, 3'UTR, transcription termination site (TTS), and non-coding). b Distribution of CpG position relative to CpG islands (CpG island, shore, shelf, and open sea) 

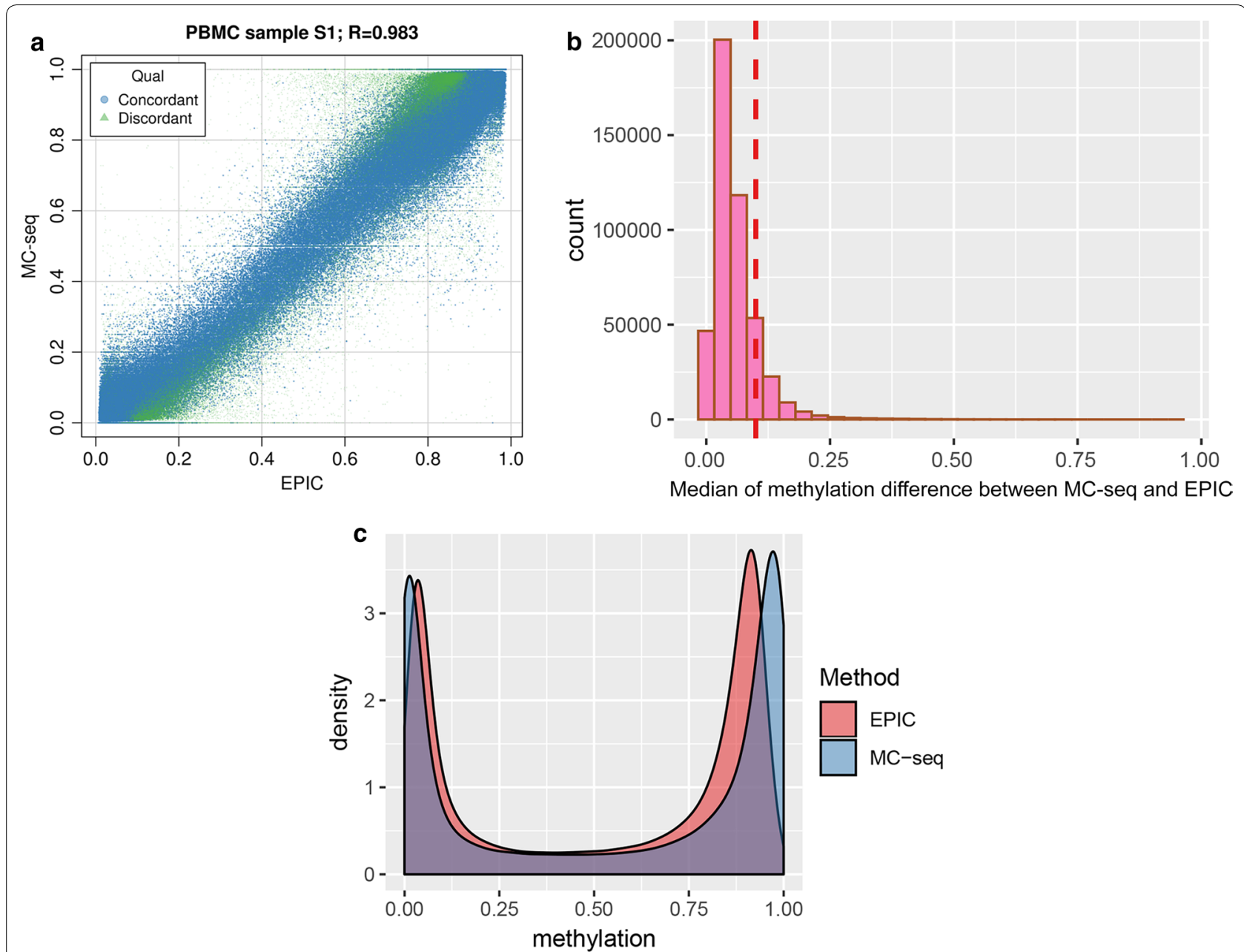

Fig. 3 Comparing methylation values among common CpG sites between MC-seq and EPIC. a Correlation of methylation values measured by MC-seq and EPIC array among common CpG sites in participant S1. Blue dots represent concordant CpGs with $\Delta \beta<0.1$ between the two platforms and green dots represent discordant quality with $\triangle \beta \geq 0.1$; $\boldsymbol{b}$ The distribution of median $\triangle \beta$ in common CpG sites between MC-seq and EPIC array. The red dotted line represents $\Delta \beta=0.1$ as a cutoff for concordant CpG site between two platforms. $\mathbf{c}$ The density plot of methylation values among common CpG sites profiled by MC-seq and EPIC array in participant S1

than the EPIC array; however, the proportion of CpG sites mapped on functional genomic regions was similar between the two platforms. Methylation at a majority of CpG sites between the two platforms was highly correlated, while methylation at a low percentage of CpG sites differed significantly between the two platforms. Specifically, we found that methylation at $239 \mathrm{CpG}$ sites differed significantly between the two platforms with absolute $\Delta \beta$ values greater than 0.5 , which suggests that these $\mathrm{CpG}$ sites should be interpreted with caution in EWAS studies.

Our results show that MC-seq produces highly reliable CpG site methylation estimates across the genome. The observed CpG-based reproducibility is high, suggesting that technical variation on $\mathrm{CpG}$ calls is low. Inter-personal methylation variation is important for EWAS analysis. We found that our participant-based methylation on common CpG sites across four participants is also highly correlated, which further demonstrates the high reproducibility of this platform.

One disadvantage of sequencing-based approaches is the requirement for a larger quantity of input DNA than array-based approaches for methylation profiling. The recommended input DNA for Agilent SureSelect platform is 1ug, while input DNA quantity for EPIC array can be as 
low as $250 \mathrm{ng}$. Input DNA quantity is one important consideration influencing study design and methylation assay platform selection for population-based EWAS. Agilent has reported that DNA quantity can be as low as $250 \mathrm{ng}$ for SureSelect sequencing [14]. To examine whether DNA quantity impacts the performance of MC-seq and to test whether low input DNA quantity also produces reliable CpG detection, we compared the capacity of CpG site detection across three different DNA input quantities. We found that medium DNA input quantity (i.e., $300 \mathrm{ng}$ to $1000 \mathrm{ng}$ ) reliably detected CpG sites is comparable to the number of $\mathrm{CpG}$ sites captured by high DNA input quantity (i.e., greater than $1000 \mathrm{ng}$ ). Low DNA input quantity (i.e., less than $300 \mathrm{ng}$ ) detected the lowest number of $\mathrm{CpG}$ sites compared with high and medium DNA input quantity. For samples with low DNA input quantity, additional PCR cycles are needed to ensure post-capture library yield that results in extensive duplicate reads. In the four low DNA input samples, the duplicate rate exceeds $80 \%$. Thus, removing duplicate reads is an important step in the QC process for $\mathrm{MC}$-seq. We found that the number of $\mathrm{CpG}$ sites in low DNA input samples without duplicated reads still is significantly higher than the number of $\mathrm{CpG}$ sites detected by the EPIC array.

Consistent with previous reports, we found that methylation at the majority of CpG sites measured by both approaches $(>98 \%)$ is highly consistent between MC-seq and array-based methods. However, we identified $1.4 \%$ of CpG sites with discrepancies in CpG methylation that exceeds $10 \%$. More importantly, 239 out of 60,753 discordant CpG sites had methylation differences exceeding $50 \%$. These CpG sites are located on 159 gene regions (Table 4). Some of these genes have been previously reported to be associated with diseases. For example, SLC45A4 was reported to harbor an epigenetic marker for adiposity [21]. The methylation $\beta$ differs on the CpG site of this gene by as much as 0.63 between the two platforms. We have also identified those CpG sites that showed less but still apparent discrepancy between the two assay platforms (i.e., absolute difference of beta values between 0.1 and 0.5 ). The top 100 CpG sites discrepant in a range of $0.1-0.4$ between two platforms are presented in Table $\mathrm{S} 2$ to allow investigators to consider this potential source of bias in EWAS findings. The discrepancy might be due to bias in the performance of the beadchip assay at these positions, sequence context-dependent impacts on the performance of sequencing, batch effects, or a combination of these possibilities. This large discrepancy warrants further investigation and interpretation of findings at these $\mathrm{CpG}$ sites must be interpreted with caution.

One of the limitations of this study is the small number of participants used to estimate inter-sample variability. A previous study used a benchmark approach to evaluate performance of different platforms [17] and concluded that the EPIC array performed better than the MC-seq platform. However, the study did not remove duplicate reads as part of their data processing, which may have compromised the $\mathrm{QC}$ for MC-seq data processing as discussed above. Future studies, including benchmarking using a larger sample size, could further improve the analysis of platform performance. Of note, MCseq detected high percentages of $\mathrm{CHG}$ and $\mathrm{CHH}$ sites across four methylome, which is consistent with previous reports [15]. The significances of those methylation sites warrant further investigation.

New approaches to measurement of DNA methylation continue to emerge that may warrant similar investigation in an ongoing effort to provide users with empiric comparisons to inform decisions about platform selection. One recent approach is enzymatic methylsequencing (EM-seq) (e.g., NEBNext EM-seq by New England Biolabs, Ipswich, MA) [22]. The input genomic DNA requirement is low 10-200 ng and EM-seq has comparable performance to WGBS [22], but its performance in relation to array- or capture sequencing-based approaches has not been reported. Should EM-seq gain popularity, it would be important to directly compare the performance of MC-seq and EM-seq to provide empiric evidence to users to inform platform selection.

Nevertheless, we have demonstrated that MC-seq is an efficient, reliable, and affordable platform that allows medium input quantity of DNA input (i.e., $>300 \mathrm{ng}$ ), which is equivalent to DNA input required for EPIC array. $\mathrm{MC}$-seq has the advantage of capturing significantly more $\mathrm{CpG}$ sites than the EPIC array. Although methylation measurements between the two platforms are highly consistent, we have identified a small number of CpG sites that must be interpreted with caution if they are associated with a trait of interest because they showed significant discrepancies between the two platforms. 
Table 4 Discordant CpG sites between MC-seq and EPIC (difference>0.5)

\begin{tabular}{|c|c|c|c|c|c|c|c|c|}
\hline Probe & Chr & Position & Gene & MC-seq median & EPIC median & $\begin{array}{l}\text { Median Difference } \\
\text { between MC-seq } \\
\text { and EPIC }\end{array}$ & Refgene group & $\begin{array}{l}\text { Relation } \\
\text { to CpG } \\
\text { island }\end{array}$ \\
\hline cg09156519 & 9 & 103361572 & & 0.009 & 0.960 & 0.95 & & S_Shore \\
\hline cg18176117 & 9 & 96097296 & C9orf129 & 0.000 & 0.932 & 0.93 & Body & N_Shore \\
\hline cg14268958 & 10 & 133453066 & & 0.000 & 0.899 & 0.89 & & S_Shelf \\
\hline cg10576280 & 10 & 124133822 & PLEKHA1 & 0.072 & 0.948 & 0.88 & TSS1500 & N_Shore \\
\hline cg01005486 & 3 & 13246006 & & 0.047 & 0.886 & 0.84 & & Island \\
\hline cg23433318 & 19 & 667542 & & 0.007 & 0.866 & 0.84 & & Island \\
\hline cg10766172 & 7 & 27498479 & & 0.989 & 0.147 & 0.82 & & \\
\hline cg11812439 & 4 & 68928706 & $\begin{array}{l}\text { LOC550113;SYT14P1;T } \\
\text { MPRSS11F }\end{array}$ & 0.973 & 0.155 & 0.82 & Body; Body; Body & \\
\hline cg23950473 & 5 & 154393265 & $K I F 4 B ; K I F 4 B$ & 0.992 & 0.174 & 0.82 & 1stExon; 5'UTR & \\
\hline cg00259849 & 8 & 4183880 & CSMD1 & 0.000 & 0.821 & 0.82 & Body & \\
\hline cg23981150 & 1 & 161111090 & & 1.000 & 0.217 & 0.78 & & Island \\
\hline cg09698465 & 12 & 133000178 & & 0.906 & 0.080 & 0.78 & & Island \\
\hline cg20450977 & 11 & 10529463 & MTRNR2L8; MTRNR2L8 & 0.964 & 0.189 & 0.77 & 3'UTR; 1stExon & \\
\hline cg01053463 & 1 & 26186087 & Clorf135 & 0.021 & 0.757 & 0.76 & TSS1500 & Island \\
\hline cg12499827 & 2 & 202004893 & $\begin{array}{l}\text { CFLAR; CFLAR; CFLAR; } \\
\text { CFLAR; CFLAR; } \\
\text { CFLAR;CFLAR; } \\
\text { CFLAR; CFLAR }\end{array}$ & 0.971 & 0.215 & 0.76 & $\begin{array}{l}\text { TSS200; Body; Body; } \\
\text { Body; Body; Body; } \\
\text { Body; Body; Body }\end{array}$ & \\
\hline cg03133777 & 2 & 170361364 & BBS5 & 1.000 & 0.244 & 0.75 & 3'UTR & \\
\hline cg19040702 & 17 & 22023833 & & 0.969 & 0.230 & 0.75 & & \\
\hline cg21675871 & 11 & 69813397 & & 0.217 & 0.960 & 0.74 & & Island \\
\hline cg04240493 & 3 & 148414664 & $\begin{array}{l}\text { AGTR1; AGTR1; AGTR1; } \\
\quad \text { AGTR1 }\end{array}$ & 0.979 & 0.258 & 0.72 & $\begin{array}{l}\text { TSS1500; TSS1500; } \\
\text { TSS1500; TSS1500 }\end{array}$ & N_Shore \\
\hline cg16889427 & 10 & 127584375 & FANK1 & 0.036 & 0.759 & 0.71 & TSS1500 & Island \\
\hline cg25916505 & 18 & 32820654 & ZNF397; ZNF397 & 0.000 & 0.711 & 0.71 & TSS1500; TSS1500 & N_Shore \\
\hline cg13525026 & 17 & 18061071 & MYO15A & 1.000 & 0.290 & 0.71 & Body & \\
\hline cg07825433 & 4 & 1215099 & CTBP1; CTBP1 & 0.000 & 0.716 & 0.71 & Body; Body & N_Shelf \\
\hline $\operatorname{cg} 03846641$ & 2 & 109746751 & $\begin{array}{l}\text { LOC100287216; } \\
\text { SH3RF3 }\end{array}$ & 0.239 & 0.952 & 0.71 & TSS200; Body & Island \\
\hline cg19188207 & 2 & 10340837 & C2orf48 & 1.000 & 0.290 & 0.71 & Body & \\
\hline cg11495544 & 17 & 73402155 & GRB2; GRB2 & 0.753 & 0.049 & 0.70 & TSS1500; TSS1500 & S_Shore \\
\hline cg06931905 & 8 & 42036940 & PLAT; PLAT & 0.896 & 0.197 & 0.70 & Body; Body & \\
\hline cg03348902 & 1 & 569603 & & 0.869 & 0.168 & 0.70 & & \\
\hline cg27120934 & 6 & 129480619 & LAMA2; LAMA2 & 0.979 & 0.297 & 0.69 & Body; Body & \\
\hline cg07576219 & 1 & 55012408 & ACOT11; ACOT11 & 0.927 & 0.250 & 0.69 & TSS1500; TSS1500 & S_Shelf \\
\hline cg08400246 & 5 & 156570642 & MED7; MED7 & 0.153 & 0.870 & 0.68 & TSS1500; TSS1500 & S_Shore \\
\hline cg27626141 & 8 & 103876469 & AZIN1; AZIN1 & 0.000 & 0.682 & 0.68 & TSS200; TSS200 & Island \\
\hline cg26688472 & 2 & 203638928 & ICAIL & 0.984 & 0.303 & 0.68 & 3'UTR & Island \\
\hline cg26101183 & 10 & 65930786 & & 0.957 & 0.279 & 0.68 & & Island \\
\hline cg27090007 & 13 & 28519388 & ATP5EP2 & 0.985 & 0.321 & 0.67 & Body & \\
\hline cg11896012 & 19 & 53696753 & ZNF665 & 0.048 & 0.700 & 0.67 & TSS200 & S_Shore \\
\hline cg02606018 & 12 & 10658281 & & 0.979 & 0.323 & 0.66 & & \\
\hline cg00438164 & 4 & 100870480 & H2AFZ; LOC256880 & 0.004 & 0.650 & 0.65 & Body; TSS1500 & Island \\
\hline cg21164300 & 9 & 136098495 & & 0.000 & 0.644 & 0.64 & & N_Shelf \\
\hline cg15891076 & 10 & 65930618 & & 0.971 & 0.328 & 0.64 & & Island \\
\hline cg05948389 & 5 & 1641924 & & 0.014 & 0.660 & 0.64 & & N_Shelf \\
\hline cg10507965 & 10 & 102107251 & $S C D ; S C D$ & 0.011 & 0.642 & 0.64 & 5'UTR; 1stExon & Island \\
\hline cg21662326 & 11 & 14521493 & COPB1; $C O P B 1 ; C O P B 1$ & 0.643 & 0.012 & 0.63 & TSS200; TSS200; TSS200 & \\
\hline
\end{tabular}


Table 4 (continued)

\begin{tabular}{|c|c|c|c|c|c|c|c|c|}
\hline Probe & Chr & Position & Gene & MC-seq median & EPIC median & $\begin{array}{l}\text { Median Difference } \\
\text { between MC-seq } \\
\text { and EPIC }\end{array}$ & Refgene group & $\begin{array}{l}\text { Relation } \\
\text { to CpG } \\
\text { island }\end{array}$ \\
\hline cg09646578 & 8 & 5019363 & & 0.310 & 0.934 & 0.63 & & \\
\hline cg24717964 & 20 & 61477008 & DPH3B; DPH3B; TCFL5 & 0.986 & 0.356 & 0.63 & 1stExon; 5'UTR; Body & \\
\hline cg07437919 & 8 & 142234483 & SLC45A4 & 0.957 & 0.313 & 0.63 & Body & N_Shore \\
\hline cg01105403 & 2 & 240723304 & & 0.050 & 0.890 & 0.63 & & \\
\hline cg20482143 & 7 & 64340804 & & 0.982 & 0.346 & 0.63 & & \\
\hline $\operatorname{cg} 11187452$ & 22 & 49698612 & & 0.017 & 0.653 & 0.63 & & Island \\
\hline cg24504954 & 3 & 61237217 & FHIT; FHIT & 0.017 & 0.649 & 0.62 & TSS200; TSS200 & Island \\
\hline $\operatorname{cg} 27434351$ & 11 & 14521491 & COPB1; $C O P B 1 ; C O P B 1$ & 0.639 & 0.016 & 0.62 & TSS200; TSS200; TSS200 & \\
\hline $\operatorname{cg} 15864074$ & 2 & 120974042 & & 0.976 & 0.354 & 0.62 & & \\
\hline cg00913521 & 12 & 89893799 & WDR51B & 0.977 & 0.339 & 0.62 & Body & \\
\hline cg27534567 & 1 & 568536 & & 0.834 & 0.262 & 0.62 & & \\
\hline cg24515136 & 17 & 49024834 & & 0.949 & 0.328 & 0.62 & & S_Shelf \\
\hline cg01417615 & 1 & 52456419 & $R A B 3 B$ & 0.629 & 0.015 & 0.61 & TSS200 & Island \\
\hline cg00236302 & 12 & 69004867 & $R A P 1 B ; R A P 1 B$ & 0.000 & 0.612 & 0.61 & $5^{\prime} U T R ; 5^{\prime} U T R$ & Island \\
\hline cg10747603 & 22 & 29197018 & $X B P 1 ; X B P 1$ & 0.022 & 0.627 & 0.61 & TSS1500; TSS1500 & S_Shore \\
\hline cg03594447 & 1 & 20359744 & & 1.000 & 0.358 & 0.61 & & \\
\hline cg23045277 & 4 & 190587808 & & 0.299 & 0.910 & 0.61 & & \\
\hline cg02218809 & 16 & 29973300 & TMEM219; TMEM219 & 0.020 & 0.612 & 0.61 & TSS200; TSS200 & Island \\
\hline cg05646491 & 10 & 135379754 & SYCE1; SYCE1; SYCE1 & 0.988 & 0.382 & 0.60 & $\begin{array}{l}\text { TSS1500; 5'UTR; } \\
\text { TSS1500 }\end{array}$ & Island \\
\hline cg07596174 & 20 & 55926107 & RAE1; RAE1 & 0.014 & 0.613 & 0.60 & TSS1500; TSS200 & N_Shore \\
\hline cg03543448 & 16 & 4384967 & GLIS2 & 0.927 & 0.315 & 0.60 & Body & \\
\hline cg25793197 & 5 & 31923469 & PDZD2 & 0.976 & 0.379 & 0.60 & Body & \\
\hline cg21392229 & 2 & 161223778 & RBMS1; RBMS1 & 1.000 & 0.384 & 0.60 & Body; Body & \\
\hline cg05607320 & 12 & 53342553 & KRT18; KRT18 & 0.064 & 0.651 & 0.60 & TSS200;TSS1500 & N_Shore \\
\hline cg13896861 & 9 & 94878241 & SPTLC1; SPTLC1 & 0.117 & 0.711 & 0.60 & TSS1500; TSS1500 & S_Shore \\
\hline cg03064900 & 4 & 190566141 & & 0.323 & 0.921 & 0.60 & & N_Shore \\
\hline cg16199859 & 3 & 75263685 & & 0.861 & 0.276 & 0.60 & & \\
\hline cg15006843 & 1 & 205720633 & NUCKS1 & 0.880 & 0.260 & 0.60 & TSS1500 & S_Shore \\
\hline cg02498218 & 4 & 26361371 & $R B P J ; R B P J ; R B P J ; R B P J$ & 0.979 & 0.388 & 0.59 & $\begin{array}{l}\text { Body; Body; 5'UTR; } \\
\text { Body }\end{array}$ & Island \\
\hline cg07116712 & 15 & 96887959 & & 0.091 & 0.681 & 0.59 & & Island \\
\hline cg11643306 & 20 & 34204831 & SPAG4 & 0.038 & 0.630 & 0.59 & Body & S_Shore \\
\hline cg08568561 & 7 & 42834498 & & 0.981 & 0.392 & 0.59 & & \\
\hline cg06669598 & 6 & 127622363 & $\begin{array}{l}\text { ECHDC1; ECHDC1; } \\
\text { ECHDC1; ECHDC1; } \\
\text { ECHDC1 }\end{array}$ & 0.984 & 0.351 & 0.59 & $\begin{array}{l}\text { 3'UTR; 3'UTR; Body; } \\
\text { Body; Body }\end{array}$ & \\
\hline cg22805431 & 3 & 113955600 & ZNF80 & 0.983 & 0.409 & 0.59 & 1stExon & \\
\hline cg24636332 & 17 & 4437925 & SPNS2 & 0.301 & 0.939 & 0.59 & Body & N_Shore \\
\hline cg05924191 & 15 & 35279830 & ZNF770 & 0.008 & 0.605 & 0.59 & $5^{\prime} U T R$ & N_Shore \\
\hline $\operatorname{cg} 14402194$ & 14 & 23398944 & $\begin{array}{l}\text { PRMT5; PRMT5; } \\
\text { PRMT5; PRMT5; } \\
\text { PRMT5; PRMT5; } \\
\text { LOC101926933 }\end{array}$ & 0.028 & 0.592 & 0.58 & $\begin{array}{l}\text { TSS200; TSS200; } \\
\text { TSS200; TSS200; } \\
\text { TSS200; TSS200; Body }\end{array}$ & S_Shore \\
\hline cg01737532 & 4 & 190862170 & $F R G 1$ & 0.000 & 0.584 & 0.58 & 1stExon & Island \\
\hline cg25744017 & 15 & 52819324 & MYO5A; MYO5A & 0.957 & 0.379 & 0.58 & Body;Body & N_Shore \\
\hline cg03432151 & 15 & 89745000 & ABHD2; ABHD2 & 0.948 & 0.360 & 0.58 & 3'UTR; 3'UTR & \\
\hline cg27196695 & 10 & 134571377 & INPP5A & 1.000 & 0.420 & 0.58 & Body & \\
\hline cg27571351 & 10 & 17619364 & & 0.986 & 0.407 & 0.58 & & \\
\hline cg02775804 & 2 & 120974080 & & 0.977 & 0.401 & 0.58 & & \\
\hline
\end{tabular}


Table 4 (continued)

\begin{tabular}{|c|c|c|c|c|c|c|c|c|}
\hline Probe & Chr & Position & Gene & MC-seq median & EPIC median & $\begin{array}{l}\text { Median Difference } \\
\text { between MC-seq } \\
\text { and EPIC }\end{array}$ & Refgene group & $\begin{array}{l}\text { Relation } \\
\text { to CpG } \\
\text { island }\end{array}$ \\
\hline $\operatorname{cg} 16461530$ & 10 & 134798264 & & 0.664 & 0.106 & 0.58 & & \\
\hline cg12654770 & 10 & 52487693 & & 0.962 & 0.385 & 0.58 & & \\
\hline cg16112880 & 1 & 201123745 & TMEM9 & 0.003 & 0.579 & 0.58 & TSS200 & Island \\
\hline cg20641423 & 8 & 125315065 & & 0.911 & 0.338 & 0.57 & & S_Shore \\
\hline cg23248615 & 10 & 2005709 & & 0.905 & 0.313 & 0.57 & & \\
\hline cg25550279 & 7 & 53254983 & & 0.965 & 0.381 & 0.57 & & Island \\
\hline cg01070250 & 1 & 569687 & & 0.843 & 0.271 & 0.57 & & \\
\hline cg06977575 & 4 & 139481990 & & 0.953 & 0.376 & 0.57 & & Island \\
\hline cg14511644 & 9 & 15055021 & & 0.977 & 0.399 & 0.57 & & \\
\hline cg08947542 & 8 & 35383200 & UNC5D & 0.879 & 0.309 & 0.57 & Body & \\
\hline cg10258063 & 2 & 217363243 & RPL37A & 0.043 & 0.613 & 0.57 & TSS1500 & N_Shore \\
\hline cg24209723 & 18 & 12913133 & & 0.973 & 0.399 & 0.57 & & S_Shore \\
\hline cg02265379 & 5 & 87898506 & LOC645323 & 0.971 & 0.384 & 0.57 & Body & Island \\
\hline cg18925601 & 7 & 158752715 & & 0.006 & 0.574 & 0.57 & & Island \\
\hline cg01406075 & 11 & 58731104 & & 0.885 & 0.309 & 0.57 & & N_Shore \\
\hline cg13545297 & 12 & 54404315 & HOXC8 & 0.229 & 0.791 & 0.57 & Body & S_Shore \\
\hline cg09036531 & 10 & 96991505 & & 0.968 & 0.402 & 0.57 & & \\
\hline cg25649283 & 9 & 140714075 & EHMT1 & 0.382 & 0.950 & 0.57 & Body & Island \\
\hline cg06204030 & 17 & 7792051 & CHD3; CHD3; CHD3 & 0.761 & 0.141 & 0.57 & TSS200; TSS200; Body & S_Shelf \\
\hline cg18627328 & 19 & 621561 & POLRMT & 0.980 & 0.411 & 0.56 & Body & Island \\
\hline $\operatorname{cg} 13085681$ & 8 & 48920761 & UBE2V2 & 0.009 & 0.576 & 0.56 & TSS1500 & N_Shore \\
\hline cg00999469 & 6 & 25107287 & CMAHP & 0.043 & 0.931 & 0.56 & Body & \\
\hline cg20960039 & 9 & 130213605 & $\begin{array}{l}\text { LRSAM1; RPL12; } \\
\text { LRSAM1; LRSAM1; } \\
\text { LRSAM1; RPL12 }\end{array}$ & 0.020 & 0.582 & 0.56 & $\begin{array}{l}\text { TSS1500; 1stExon; } \\
\text { TSS200; TSS200; } \\
\text { TSS200; 5'UTR }\end{array}$ & Island \\
\hline cg04400841 & 2 & 208988863 & CRYGD & 0.215 & 0.764 & 0.56 & Body & Island \\
\hline $\operatorname{cg} 12476298$ & 19 & 58426697 & ZNF417 & 0.977 & 0.407 & 0.56 & Body & N_Shore \\
\hline cg23997402 & 19 & 14275669 & LPHN1; LPHN1 & 0.972 & 0.442 & 0.56 & Body; Body & S_Shore \\
\hline cg16935370 & 5 & 154393281 & KIF $4 B ; K I F 4 B$ & 0.981 & 0.414 & 0.56 & 1stExon; 5'UTR & \\
\hline cg04222159 & 1 & 204981786 & $\begin{array}{l}\text { NFASC; NFASC; NFASC; } \\
\quad \text { NFASC }\end{array}$ & 0.630 & 0.073 & 0.56 & $\begin{array}{l}\text { Body; Body; Body; } \\
\text { Body }\end{array}$ & \\
\hline cg06396237 & 8 & 120779442 & TAF2 & 0.985 & 0.430 & 0.56 & Body & \\
\hline cg06599543 & 6 & 165749446 & PDE10A;PDE10A & 0.857 & 0.332 & 0.56 & Body;Body & S_Shore \\
\hline cg11566832 & 10 & 88659593 & BMPR1A & 0.145 & 0.693 & 0.55 & Body & \\
\hline cg20334010 & 15 & 41047916 & RMDN3; RMDN3 & 0.020 & 0.570 & 0.55 & TSS1500; TSS1500 & S_Shore \\
\hline cg18245781 & 5 & 3659697 & & 0.283 & 0.852 & 0.55 & & \\
\hline cg03761810 & 2 & 10264850 & RRM2; RRM2 & 0.018 & 0.567 & 0.55 & Body; Body & S_Shore \\
\hline cg02122372 & 3 & 149657597 & RNF13; RNF13 & 1.000 & 0.439 & 0.55 & Body; Body & \\
\hline cg06753227 & 18 & 9475508 & RALBP1 & 0.000 & 0.553 & 0.55 & TSS200 & Island \\
\hline cg14131834 & 13 & 45914250 & LOC100190939; TPT1 & 0.040 & 0.594 & 0.55 & TSS1500; Body & N_Shore \\
\hline cg25583180 & 5 & 177614382 & GMCL1L & 1.000 & 0.451 & 0.55 & Body & Island \\
\hline cg09112623 & 6 & 33756905 & LEMD2; LEMD2 & 0.568 & 0.019 & 0.55 & 5'UTR; 1stExon & Island \\
\hline cg11759477 & 4 & 190861959 & FRG1 & 0.000 & 0.548 & 0.55 & TSS200 & Island \\
\hline $\operatorname{cg} 12796755$ & 14 & 51132292 & SAV1 & 0.932 & 0.383 & 0.55 & Body & N_Shelf \\
\hline cg19693446 & 14 & 102144192 & & 0.958 & 0.407 & 0.55 & & \\
\hline cg25187648 & 3 & 49395165 & GPX1;GPX1;GPX1 & 0.018 & 0.568 & 0.55 & Body; 3'UTR; 1stExon & Island \\
\hline cg20391833 & 6 & 167116208 & RPS6KA2 & 0.964 & 0.431 & 0.55 & Body & \\
\hline cg09705232 & 6 & 97611802 & MIR548H3; C6orf167 & 0.974 & 0.428 & 0.55 & Body; Body & \\
\hline cg04643437 & 12 & 14518655 & ATF7IP; ATF7IP & 0.000 & 0.561 & 0.55 & 1stExon; 5'UTR & Island \\
\hline
\end{tabular}


Table 4 (continued)

\begin{tabular}{|c|c|c|c|c|c|c|c|c|}
\hline Probe & Chr & Position & Gene & MC-seq median & EPIC median & $\begin{array}{l}\text { Median Difference } \\
\text { between MC-seq } \\
\text { and EPIC }\end{array}$ & Refgene group & $\begin{array}{l}\text { Relation } \\
\text { to CpG } \\
\text { island }\end{array}$ \\
\hline cg17558062 & 13 & 45965415 & LOC100190939 & 1.000 & 0.456 & 0.54 & Body & Island \\
\hline cg26825848 & 4 & 190566175 & & 0.350 & 0.899 & 0.54 & & N_Shore \\
\hline cg13943141 & 9 & 93205862 & & 0.846 & 0.296 & 0.54 & & \\
\hline cg26951705 & 19 & 56612697 & ZNF787 & 0.000 & 0.542 & 0.54 & Body & Island \\
\hline cg24654094 & 1 & 160340832 & NHLH1 & 0.964 & 0.433 & 0.54 & Body & Island \\
\hline cg02996355 & 14 & 81879375 & & 0.909 & 0.364 & 0.54 & & \\
\hline cg11914812 & 12 & 56904792 & & 1.000 & 0.459 & 0.54 & & \\
\hline cg24895977 & 19 & 35861796 & & 0.990 & 0.450 & 0.54 & & \\
\hline cg11637682 & 6 & 147124984 & LOC729176; C6orf103 & 0.867 & 0.300 & 0.54 & TSS200; Body & \\
\hline cg07089633 & 14 & 73396378 & $\begin{array}{l}\text { DCAF4; DCAF4; } \\
\text { DCAF4; DCAF4; } \\
\text { DCAF4 }\end{array}$ & 1.000 & 0.450 & 0.54 & $\begin{array}{l}\text { 5'UTR; 5'UTR; 5'UTR; } \\
\text { 5'UTR; 5'UTR }\end{array}$ & S_Shelf \\
\hline cg20360416 & 4 & 7246127 & SORCS2 & 0.018 & 0.659 & 0.54 & Body & \\
\hline cg25627920 & 17 & 39992620 & $\begin{array}{l}\text { NT5C3B; NT5C3B; } \\
\text { NT5C } 3 B ; K L H L 10\end{array}$ & 0.016 & 0.552 & 0.54 & $\begin{array}{l}\text { TSS200; TSS200; } \\
\text { TSS200; TSS1500 }\end{array}$ & Island \\
\hline cg24000259 & 5 & 55488291 & ANKRD55 & 0.961 & 0.411 & 0.54 & Body & \\
\hline cg09138437 & 11 & 64527189 & PYGM; PYGM & 0.993 & 0.445 & 0.54 & 1stExon; 1stExon & \\
\hline cg02673636 & 1 & 109647056 & & 0.976 & 0.437 & 0.54 & & S_Shelf \\
\hline cg18740872 & 5 & 39220260 & $F Y B ; F Y B$ & 1.000 & 0.466 & 0.53 & TSS1500; TSS1500 & \\
\hline cg14354292 & 7 & 63353606 & & 0.960 & 0.426 & 0.53 & & \\
\hline cg17704839 & 19 & 9939038 & UBL5; UBL5 & 0.014 & 0.542 & 0.53 & Body; Body & S_Shore \\
\hline cg05971373 & 7 & 157498604 & $\begin{array}{l}\text { PTPRN2; PTPRN2; } \\
\text { PTPRN2 }\end{array}$ & 1.000 & 0.467 & 0.53 & Body; Body; Body & S_Shelf \\
\hline cg05291429 & 17 & 1494566 & SLC43A2 & 0.402 & 0.969 & 0.53 & Body & S_Shelf \\
\hline cg08841342 & 3 & 156528470 & PA2G4P4 & 0.976 & 0.444 & 0.53 & Body & \\
\hline cg04096697 & 6 & 37012867 & & 0.983 & 0.451 & 0.53 & & Island \\
\hline cg26878995 & 1 & 168106731 & GPR161 & 0.052 & 0.570 & 0.53 & TSS1500 & S_Shore \\
\hline cg24031524 & 20 & 19804606 & & 0.990 & 0.468 & 0.53 & & \\
\hline cg19311470 & 4 & 39460490 & $R P L 9 ; R P L 9 ; L I A S ; L I A S$ & 0.004 & 0.529 & 0.53 & $\begin{array}{l}\text { TSS1500; 5'UTR; TSS200; } \\
\text { TSS200 }\end{array}$ & Island \\
\hline cg02181482 & 5 & 178942685 & & 0.956 & 0.449 & 0.53 & & \\
\hline cg05346902 & 19 & 47910374 & MEIS3; MEIS3 & 0.068 & 0.593 & 0.53 & Body; Body & Island \\
\hline cg16470772 & 10 & 8203304 & & 0.971 & 0.445 & 0.53 & & \\
\hline cg10115022 & 1 & 27527942 & & 0.974 & 0.464 & 0.53 & & Island \\
\hline cg27231717 & 6 & 26319377 & & 0.905 & 0.386 & 0.53 & & \\
\hline cg09451549 & 19 & 8386408 & RPS28; NDUFA7; RPS28 & 0.000 & 0.527 & 0.53 & 5'UTR; TSS200; 1stExon & Island \\
\hline cg07628841 & 2 & 27851430 & $\begin{array}{l}\text { GPN1; CCDC121; } \\
\text { GPN1; GPN1; } \\
\text { CCDC121; CCDC121; } \\
\text { GPN1; GPN1 }\end{array}$ & 0.010 & 0.536 & 0.53 & $\begin{array}{l}\text { TSS200; 1stExon; } \\
\text { TSS200; TSS1500; } \\
\text { 5'UTR; 1stExon; } \\
\text { TSS200; TSS1500 }\end{array}$ & \\
\hline cg03816081 & 10 & 29577743 & LYZL1 & 0.863 & 0.301 & 0.52 & TSS1500 & \\
\hline cg00762003 & 21 & 45393541 & AGPAT3; AGPAT3 & 0.383 & 0.892 & 0.52 & Body; Body & Island \\
\hline cg19466922 & 7 & 130138026 & $\begin{array}{l}\text { MEST;MEST; MEST; } \\
\text { MEST;MEST;MEST }\end{array}$ & 1.000 & 0.476 & 0.52 & $\begin{array}{l}\text { Body; Body; Body; } \\
\text { Body; Body; Body }\end{array}$ & \\
\hline cg07712165 & 17 & 80899280 & $T B C D$ & 0.440 & 0.959 & 0.52 & Body & Island \\
\hline cg01199952 & 13 & 25591486 & & 0.984 & 0.456 & 0.52 & & N_Shore \\
\hline cg11374834 & 3 & 75263691 & & 0.950 & 0.428 & 0.52 & & \\
\hline cg02974491 & 1 & 1162280 & SDF4; SDF4 & 0.403 & 0.964 & 0.52 & Body; Body & Island \\
\hline cg10555853 & 1 & 33516627 & & 0.929 & 0.407 & 0.52 & & Island \\
\hline cg21216606 & 2 & 207275704 & & 0.985 & 0.464 & 0.52 & & \\
\hline
\end{tabular}


Table 4 (continued)

\begin{tabular}{|c|c|c|c|c|c|c|c|c|}
\hline Probe & Chr & Position & Gene & MC-seq median & EPIC median & $\begin{array}{l}\text { Median Difference } \\
\text { between MC-seq } \\
\text { and EPIC }\end{array}$ & Refgene group & $\begin{array}{l}\text { Relation } \\
\text { to CpG } \\
\text { island }\end{array}$ \\
\hline cg17711541 & 6 & 26124704 & $\begin{array}{l}\text { HIST1H2AC; } \\
\quad \text { HIST1H2BC }\end{array}$ & 0.007 & 0.529 & 0.52 & 1stExon; TSS1500 & Island \\
\hline cg06412823 & 7 & 22541074 & STEAP1B; STEAP1B & 0.196 & 0.739 & 0.52 & TSS1500; TSS1500 & S_Shore \\
\hline cg03054343 & 11 & 50238214 & & 1.000 & 0.463 & 0.52 & & Island \\
\hline cg05766605 & 1 & 19384827 & & 0.423 & 0.938 & 0.52 & & \\
\hline cg07684215 & 10 & 132976057 & TCERG1L & 0.181 & 0.923 & 0.52 & Body & \\
\hline cg27193858 & 6 & 41169120 & TREML2 & 0.181 & 0.691 & 0.52 & TSS200 & \\
\hline cg00964321 & 16 & 15083956 & PDXDC1 & 0.906 & 0.386 & 0.52 & Body & Island \\
\hline cg25394572 & 11 & 56457777 & OR8U8 & 0.949 & 0.429 & 0.52 & Body & \\
\hline cg10667969 & 3 & 149181941 & & 0.967 & 0.458 & 0.52 & & \\
\hline cg18394854 & 5 & 8457818 & & 0.212 & 0.732 & 0.52 & & Island \\
\hline cg05741225 & 10 & 133917303 & JAKMIP3 & 0.906 & 0.370 & 0.52 & TSS1500 & \\
\hline cg06026769 & 12 & 20704492 & PDE3A & 0.993 & 0.473 & 0.52 & Body & N_Shore \\
\hline cg09032630 & 6 & 27831956 & HIST1H2AL & 0.802 & 0.311 & 0.52 & TSS1500 & N_Shore \\
\hline cg16626480 & 22 & 25575426 & KIAA1671 & 0.950 & 0.422 & 0.52 & Body & Island \\
\hline cg24534731 & 17 & 36888147 & CISD3 & 0.969 & 0.457 & 0.52 & Body & S_Shore \\
\hline cg16202259 & 14 & 104625420 & KIF26A & 0.058 & 0.962 & 0.52 & Body & Island \\
\hline cg25325592 & 8 & 1439535 & & 0.408 & 0.923 & 0.52 & & N_Shore \\
\hline cg00391025 & 3 & 100427239 & TFG; TFG & 1.000 & 0.477 & 0.52 & TSS1500; TSS1500 & N_Shore \\
\hline cg25149037 & 17 & 39736213 & & 0.820 & 0.454 & 0.52 & & \\
\hline cg19120749 & 11 & 1431650 & $\begin{array}{l}\text { BRSK2; BRSK2; BRSK2; } \\
\text { BRSK2; BRSK2; BRSK2 }\end{array}$ & 0.468 & 0.983 & 0.52 & $\begin{array}{l}\text { TSS1500; TSS200; Body; } \\
\text { Body; Body; Body }\end{array}$ & Island \\
\hline cg24270624 & 10 & 95721318 & PIPSL & 0.947 & 0.421 & 0.52 & Body & \\
\hline cg16346588 & 10 & 242978 & $\begin{array}{l}\text { ZMYND11; ZMYND11; } \\
\text { ZMYND11 }\end{array}$ & 0.970 & 0.459 & 0.51 & Body; Body; Body & \\
\hline cg02750322 & 15 & 83673816 & $\begin{array}{l}\text { C15orf40; C15orf40; } \\
\text { C15orf40; C15orf40; } \\
\text { C15orf40 }\end{array}$ & 0.966 & 0.451 & 0.51 & $\begin{array}{l}\text { Body; 3'UTR; Body; } \\
\text { Body; Body }\end{array}$ & \\
\hline cg04363536 & 3 & 49466872 & NICN1 & 0.000 & 0.515 & 0.51 & TSS200 & S_Shore \\
\hline cg17883371 & 1 & 91359225 & & 1.000 & 0.480 & 0.51 & & Island \\
\hline cg25018832 & 1 & 564471 & LOC101928626 & 0.602 & 0.088 & 0.51 & TSS200 & \\
\hline cg16838729 & 4 & 43901032 & & 0.903 & 0.394 & 0.51 & & \\
\hline cg23222247 & 17 & 47302219 & $\begin{array}{l}\text { PHOSPHO1; PHOS- } \\
\text { PHO1 }\end{array}$ & 0.009 & 0.531 & 0.51 & Body;Body & Island \\
\hline cg19496566 & 19 & 48249018 & GLTSCR2 & 0.009 & 0.535 & 0.51 & 1stExon & Island \\
\hline cg03165426 & 7 & 30726958 & CRHR2; CRHR2 & 0.429 & 0.942 & 0.51 & Body; 5'UTR & \\
\hline cg19600494 & 2 & 106959525 & & 0.968 & 0.455 & 0.51 & & Island \\
\hline cg10854807 & 17 & 79479308 & ACTG1 & 0.004 & 0.522 & 0.51 & Body & Island \\
\hline cg20699097 & 11 & 111957680 & $\begin{array}{l}\text { TIMMBB; TIMM8B; } \\
\text { SDHD }\end{array}$ & 0.009 & 0.524 & 0.51 & $\begin{array}{l}\text { TSS200; TSS200; } \\
\text { 1stExon }\end{array}$ & Island \\
\hline cg22819767 & 10 & 11866910 & Cloorf47 & 0.958 & 0.440 & 0.51 & 5'UTR & S_Shore \\
\hline cg20254251 & 8 & 144557206 & $\mathrm{ZC} 3 \mathrm{H} 3$ & 0.993 & 0.450 & 0.51 & Body & \\
\hline cg00590830 & 1 & 32385224 & $\begin{array}{l}\text { PTP4A2; PTP4A2; } \\
\text { PTP4A2; PTP4A2; } \\
\text { PTP4A2 }\end{array}$ & 0.971 & 0.437 & 0.51 & $\begin{array}{l}\text { 1stExon; 1stExon; } \\
\text { 5'UTR; 5'UTR; 5'UTR }\end{array}$ & \\
\hline cg03877767 & 2 & 11680057 & GREB1; GREB1 & 0.172 & 0.683 & 0.51 & 5'UTR; TSS200 & \\
\hline cg00487526 & 15 & 90818384 & & 0.956 & 0.444 & 0.51 & & Island \\
\hline cg17501384 & 2 & 217364031 & RPL37A & 0.017 & 0.521 & 0.51 & Body & S_Shore \\
\hline cg17646418 & 6 & 166911767 & RPS6KA2; RPS6KA2 & 0.987 & 0.468 & 0.51 & Body; Body & \\
\hline
\end{tabular}


Table 4 (continued)

\begin{tabular}{|c|c|c|c|c|c|c|c|c|}
\hline Probe & Chr & Position & Gene & MC-seq median & EPIC median & $\begin{array}{l}\text { Median Difference } \\
\text { between MC-seq } \\
\text { and EPIC }\end{array}$ & Refgene group & $\begin{array}{l}\text { Relation } \\
\text { to CpG } \\
\text { island }\end{array}$ \\
\hline cg06757405 & 5 & 140789450 & $\begin{array}{l}\text { PCDHGA4; PCDHGA9; } \\
\text { PCDHGA1; PCD- } \\
\text { HGB1; PCDHGB6; } \\
\text { PCDHGB6; PCD- } \\
\text { HGB3; PCDHGA6; } \\
\text { PCDHGA8; PCD- } \\
\text { HGA5; PCDHGB4; } \\
\text { PCDHGA3; } \\
\text { PCDHGA2; PCD- } \\
\text { HGB2; PCDHGA7; } \\
\text { PCDHGB5 }\end{array}$ & 0.108 & 0.607 & 0.51 & $\begin{array}{l}\text { Body; Body; Body; } \\
\text { Body; } 1 \text { stExon; } \\
\text { 1stExon; Body; Body; } \\
\text { Body; Body; Body; } \\
\text { Body; Body; Body; } \\
\text { Body; Body }\end{array}$ & Island \\
\hline $\operatorname{cg} 13448596$ & 8 & 2031599 & MYOM2 & 0.381 & 0.887 & 0.51 & Body & \\
\hline $\operatorname{cg} 16711165$ & 11 & 111957658 & $\begin{array}{l}\text { TIMM8B; TIMM8B; } \\
\text { SDHD }\end{array}$ & 0.017 & 0.522 & 0.51 & $\begin{array}{l}\text { TSS200; TSS200; } \\
\text { 1stExon }\end{array}$ & Island \\
\hline $\operatorname{cg} 16786640$ & 4 & 3485263 & DOK7; DOK7 & 0.447 & 0.954 & 0.51 & Body; Body & N_Shore \\
\hline cg07638938 & 10 & 131348599 & MGMT & 0.986 & 0.492 & 0.51 & Body & \\
\hline cg05407710 & 8 & 143329409 & TSNAREI & 0.960 & 0.451 & 0.51 & Body & N_Shelf \\
\hline cg07869994 & 3 & 174095190 & & 0.988 & 0.482 & 0.51 & & Island \\
\hline cg07455406 & 14 & 21077527 & & 0.017 & 0.523 & 0.51 & & N_Shore \\
\hline cg04576847 & 17 & 12623611 & $\begin{array}{l}\text { MYOCD; MYOCD; } \\
\text { MYOCD; MYOCD }\end{array}$ & 0.958 & 0.447 & 0.51 & $\begin{array}{l}\text { Body; } 5^{\prime} \cup T R ; \text { Body; } \\
\text { 1stExon }\end{array}$ & \\
\hline cg15699853 & 18 & 57684747 & & 0.976 & 0.467 & 0.51 & & \\
\hline $\operatorname{cg} 11231240$ & 8 & 82434638 & & 1.000 & 0.482 & 0.51 & & Island \\
\hline cg06157924 & 4 & 942005 & TMEM175 & 0.451 & 0.957 & 0.51 & Body & S_Shore \\
\hline cg23679141 & 4 & 165118930 & MARCH1; ANP32C & 0.946 & 0.445 & 0.51 & 5'UTR; TSS200 & \\
\hline cg03053358 & 17 & 1029917 & $A B R ; A B R$ & 0.446 & 0.964 & 0.51 & Body; 5'UTR & S_Shore \\
\hline $\operatorname{cg} 13705894$ & 9 & 138305338 & & 0.978 & 0.495 & 0.51 & & S_Shore \\
\hline cg18512780 & 17 & 76117734 & TMC6; TMC6 & 0.033 & 0.529 & 0.50 & Body; Body & \\
\hline cg06307940 & 16 & 46660818 & & 0.986 & 0.487 & 0.50 & & \\
\hline cg15541008 & 5 & 95297508 & ELL2; ELL2 & 0.000 & 0.516 & 0.50 & 1stExon; 5'UTR & S_Shore \\
\hline cg19969624 & 13 & 95954210 & $\begin{array}{l}A B C C 4 ; A B C C 4 ; A B C C 4 ; \\
\quad A B C C 4\end{array}$ & 0.011 & 0.507 & 0.50 & $\begin{array}{l}\text { TSS1500; TSS1500; } \\
\text { TSS1500; TSS1500 }\end{array}$ & Island \\
\hline cg01758870 & 7 & 23719630 & $\begin{array}{l}\text { C7orf46; C7orf46; } \\
\text { C7orf46 }\end{array}$ & 0.028 & 0.545 & 0.50 & TSS200;TSS200;TSS200 & Island \\
\hline cg08323201 & 15 & 101835348 & SNRPA1 & 0.000 & 0.503 & 0.50 & 1stExon & Island \\
\hline cg21863998 & 11 & 19770288 & NAV2; NAV2; NAV2 & 0.439 & 0.905 & 0.50 & Body; Body; Body & \\
\hline $\operatorname{cg} 11471802$ & 8 & 47529015 & & 0.348 & 0.882 & 0.50 & & Island \\
\hline cg22281935 & 2 & 162934111 & & 0.982 & 0.481 & 0.50 & & S_Shelf \\
\hline cg06032540 & 15 & 43941563 & $\begin{array}{l}\text { CATSPER2; CATSPER2; } \\
\text { CATSPER2 }\end{array}$ & 1.000 & 0.500 & 0.50 & $\begin{array}{l}\text { TSS1500; TSS1500; } \\
\text { TSS1500 }\end{array}$ & Island \\
\hline $\operatorname{cg} 18761878$ & 1 & 568475 & & 0.893 & 0.401 & 0.50 & & \\
\hline
\end{tabular}

\section{Conclusions}

Our results show that MC-seq is an efficient and reliable platform for methylome profiling with a broader coverage of the methylome than the array-based platform. Although methylation measurements in majority of CpGs are highly correlated, a number of CpG sites show large discrepancy between the two platforms, which warrants further investigation and needs cautious interpretation. 


\section{Supplementary information}

Supplementary information accompanies this paper at https://doi. org/10.1186/s13072-020-00372-6.

Additional file 1: Figure S1. A Manhattan plot showing the distribution of $\triangle \beta$ between MC-seq and EPIC array in PBMC by chromosome positions. Blue line represents $\Delta \beta=0.1$ and red line represents $\Delta \beta=0.5$.

Additional file 2: Table S1. Top 100 discordant CpG sites between MCseq and EPIC array $(\triangle \beta=0.1 \sim 0.4)$.

Additional file 3: Figure S2. Comparison of methylation values measured by MC-seq and EPIC array among common CpG sites in participant S2, S3, and S4. Blue dots represent concordant CpGs with $\Delta \beta<0.1$ between the two platforms and green dots represent discordant quality with $\Delta \beta \geq 0.1$

Additional file 4: Figure S3. The density plot of methylation values among CpG sites assayed in common by MC-seq and EPIC array in participant $\mathrm{S} 2, \mathrm{~S} 3$, and $\mathrm{S} 4$

\section{Abbreviations}

EM-seq: Enzymatic methyl-seq; EPIC: Illumina Infinium MethylationEPIC Beadchip; EWAS: Epigenome-wide association study; MC-seq: Methylation capture sequencing; PBMC: Peripheral blood mononuclear cell; PCR: Polymerase chain reaction; QC: Quality control; RTA: Real-time analysis; TTS: Transcription termination site; WGBS: Whole-genome bisulfite sequencing.

\section{Acknowledgements}

The project was supported by the National Institute on Drug Abuse (R03DA039745, R01DA038632, R01DA047063, R01DA047820). The authors appreciate the support of the Yale Center of Genomic Analysis and Women's Interagency HIV Study.

\section{Authors' contributions}

CS contributed to data analysis and the first draft of manuscript. XZ contributed to data processing, quality control, analysis, and manuscript preparation. BA was involved in manuscript preparation and provided peripheral blood monocyte cells. KX contributed to study design, analytical strategies, and manuscript preparation. All the authors read and approved the final manuscript.

\section{Data availability}

All methylation data from MC-seq and EPIC platforms are deposited in GEO (GSE152922).

\section{Ethics approval and consent to participate}

The study was approved by the committee of the Human Research Subject Protection at Yale University and the Institutional Research Board Committee of the Connecticut Veteran Healthcare System. De-identifiable samples were from Women's Interagency HIV Study cohort. All participants provided written consents.

\section{Competing interests}

The authors declare that they have no competing interests.

\section{Author details}

${ }^{1}$ Department of Psychiatry, Yale School of Medicine, New Haven, CT 06516, USA. ${ }^{2}$ Connecticut Veteran Healthcare System, West Haven, CT 06515, USA.

${ }^{3}$ Bluestone Center for Clinical Research, College of Dentistry, New York University, New York 10010, USA. ${ }^{4}$ Department of Oral and Maxillofacial Surgery, College of Dentistry, Yale School of Medicine, New York University, New York 10010, USA.

Received: 4 June 2020 Accepted: 2 November 2020

Published online: 23 November 2020

\section{References}

1. Bakusic J, Schaufeli W, Claes S, Godderis L. Stress, burnout and depression: a systematic review on DNA methylation mechanisms. J Psychosom Res. 2017;92:34-44.
2. Kraiczy J, Nayak KM, Howell KJ, Ross A, Forbester J, Salvestrini C, et al. DNA methylation defines regional identity of human intestinal epithelial organoids and undergoes dynamic changes during development. Gut. 2019;68(1):49-61.

3. Lam K, Pan K, Linnekamp JF, Medema JP, Kandimalla R. DNA methylation based biomarkers in colorectal cancer: a systematic review. Biochim Biophys Acta Rev Cancer. 2016;1866(1):106-20.

4. Li M, D'Arcy C, Li X, Zhang T, Joober R, Meng X. What do DNA methylation studies tell us about depression? A systematic review. Transl Psychiatry. 2019;9(1):68.

5. Nano J, Ghanbari M, Wang W, de Vries PS, Dhana K, Muka T, et al. Epigenome-wide association study identifies methylation sites associated with liver enzymes and hepatic steatosis. Gastroenterology. 2017;153(4):1096-106.

6. Teroganova N, Girshkin L, Suter CM, Green MJ. DNA methylation in peripheral tissue of schizophrenia and bipolar disorder: a systematic review. BMC Genet. 2016;17(1):27.

7. Delpu Y, Cordelier P, Cho W, Torrisani J. DNA methylation and cancer diagnosis. Int J Mol Sci. 2013;14(7):15029-58.

8. Figueroa ME, Lugthart S, Li Y, Erpelinck-Verschueren C, Deng X, Christos PJ, et al. DNA methylation signatures identify biologically distinct subtypes in acute myeloid leukemia. Cancer Cell. 2010;17(1):13-27.

9. Holm K, Hegardt C, Staaf J, Vallon-Christersson J, Jönsson G, Olsson H, et al. Molecular subtypes of breast cancer are associated with characteristic DNA methylation patterns. Breast Cancer Res. 2010;12(3):R36.

10. Berdasco M, Esteller M. Clinical epigenetics: seizing opportunities for translation. Nat Rev Genet. 2019;20(2):109-27.

11. Mohammad HP, Barbash O, Creasy CL. Targeting epigenetic modifications in cancer therapy: erasing the roadmap to cancer. Nat Med. 2019;25(3):403-18.

12. Dedeurwaerder S, Defrance $M$, Bizet M, Calonne E, Bontempi G, Fuks F. A comprehensive overview of Infinium HumanMethylation450 data processing. Brief Bioinform. 2013;15(6):929-41.

13. Pidsley R, Zotenko E, Peters TJ, Lawrence MG, Risbridger GP, Molloy $P$, et al. Critical evaluation of the Illumina MethylationEPIC BeadChip microarray for whole-genome DNA methylation profiling. Genome Biol. 2016;17(1):1-17.

14. Wang JZ, Pabon C, Napier M. Agilent SureSelectXT Methyl-Seq applications with low-input DNA and smaller capture libraries. 2017. https:// www.agilent.com/cs/library/applications/5991-7838EN.pdf.

15. Teh AL, Pan H, Lin X, Lim Yl, Patro CP, Cheong CY, et al. Comparison of methyl-capture sequencing vs. Infinium $450 \mathrm{~K}$ methylation array for methylome analysis in clinical samples. Epigenetics. 2016;11(1):36-48.

16. Sun Z, Cunningham J, Slager S, Kocher JP. Base resolution methylome profiling: considerations in platform selection, data preprocessing and analysis. Epigenomics. 2015;7(5):813-28.

17. Heiss JA, Brennan KJ, Baccarelli AA, Tellez-Rojo MM, Estrada-Gutierrez G, Wright RO, et al. Battle of epigenetic proportions: comparing Illumina's EPIC methylation microarrays and TruSeq targeted bisulfite sequencing. Epigenetics. 2020;15(1-2):174-82.

18. Wreczycka K, Gosdschan A, Yusuf D, Gruning B, Assenov Y, Akalin A. Strategies for analyzing bisulfite sequencing data. J Biotechnol. 2017:261:105-15.

19. Krueger F, Andrews SR. Bismark: a flexible aligner and methylation caller for Bisulfite-Seq applications. Bioinformatics. 2011;27(11):1571-2.

20. Zhang X, Hu Y, Justice AC, Li B, Wang Z, Zhao H, et al. DNA methylation signatures of illicit drug injection and hepatitis $C$ are associated with HIV frailty. Nat Commun. 2017;8(1):2243.

21. Lillycrop KA, Garratt ES, Titcombe P, Melton PE, Murray RJ, Barton SJ, et al. Differential SLC6A4 methylation: a predictive epigenetic marker of adiposity from birth to adulthood. Int J Obes. 2019;43(5):974-88.

22. Williams L, Bei Y, Church HE, Dai N, Dimalanta ET, Ettwiller LM, et al. Enzymatic Methyl-seq: the next generation of methylome analysis. NEB Expressions. 2019;.

\section{Publisher's Note}

Springer Nature remains neutral with regard to jurisdictional claims in published maps and institutional affiliations. 